Check for updates

Cite this: RSC Adv., 2017, 7, 53002

Received 24th June 2017

Accepted 6th November 2017

DOI: $10.1039 / c 7 r a 07040 c$

rsc.li/rsc-advances

\section{Thymoquinone reduces kidney damage in apolipoprotein E-deficient mice fed a high-cholesterol diet}

\author{
Zuowei Pei, (D) a Liyue Zhu, ${ }^{\text {' Yingshu Liu, }{ }^{c} \text { Nan Li, }{ }^{d} \text { Guang Yang }}{ }^{\text {e }}$ \\ and Hongyang Liu (D) *e
}

Hypercholesterolemia is a well-established risk factor for kidney injury that can lead to chronic kidney disease (CKD). Thymoquinone (TQ) is one of the most active ingredients in Nigella Sativa seeds. It has a variety of beneficial properties including anti-oxidative and anti-inflammatory activities. TQ exerts positive effects on DOX-induced nephropathy and ischaemia-reperfusion induced kidney injury in rats. The goal of this study was to investigate the possible protective effects of thymoquinone against kidney injury in apolipoprotein E-knockout $\left(\mathrm{ApoE}^{-1-}\right)$ mice. Eight-week-old male $\mathrm{ApoE}^{-1-}$ mice were randomly divided into the following 3 groups: a control group fed a normal diet (ND group), a group fed a high-cholesterol diet (HD group) and a group fed HD mixed with thymoquinone (HD + TQ group). All groups were given the diet for 8 weeks. Metabolic characteristics including total cholesterol (TC) and low-density lipoprotein-cholesterol $(\mathrm{LDL}-\mathrm{c})$ were lower in $\mathrm{ApoE}^{-1-} \mathrm{HD}+\mathrm{TQ}$ mice than in $\mathrm{ApoE}^{-1-} \mathrm{HD}$ mice. Oil-red $\mathrm{O}$ staining revealed excessive lipid deposition in the kidneys of $\mathrm{ApoE}^{-1-} \mathrm{HD}$ mice; however, it was significantly suppressed in the $A p o E^{-l-} \mathrm{HD}+\mathrm{TQ}$ mice. Lectin-like oxidized low-density lipoprotein receptor-1 (LOX-1) gene and protein expression was lower in the kidney tissues of $A p o E^{-1-} \mathrm{HD}+\mathrm{TQ}$ mice than those of ApoE ${ }^{-1-} \mathrm{HD}$ mice. Furthermore, macrophages and pro-inflammatory cytokines were lower in the kidney tissues of $\mathrm{ApoE}^{-1-} \mathrm{HD}+\mathrm{TQ}$ mice than in the $\mathrm{ApoE}^{-1-} \mathrm{HD}$ mice. These results indicate that thymoquinone may be a potential therapeutic agent for kidney damage from hypercholesterolemia.

\section{Introduction}

$\mathrm{ApoE}^{-1-}$ mice are a well-accepted model of hypercholesterolemia, and they have been extensively used to study the effects of this disease on atherosclerosis and renal injury. ${ }^{\mathbf{1}}$ One of our recent studies showed that in $\mathrm{ApoE}^{-1-}$ mice, dyslipidaemiarelated kidney injury is associated with marked pathological alterations, including lipid deposition, pro-inflammatory gene expression and macrophage accumulation. ${ }^{2}$ Increasing evidence has shown that lipid accumulation in the kidney contributes to the progression of chronic kidney disease (CKD)., ${ }^{3,4}$ This process is enhanced in the presence of elevated levels of plasma lipids. Macrophages phagocytose oxidized

${ }^{a}$ Department of Cardiology, Affiliated Zhongshan Hospital of Dalian University, No. 6 Jiefang Street, Dalian, China

${ }^{b}$ Rehabilitation Center, Zhejiang Hospital, 12 Lingyin Road, Hangzhou, Zhejiang, China

'Department of Endocrinology Dalian Municipal Central Hospital, 42 Xuegong Road, Dalian, China

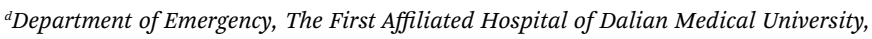
193 Lianhe Road, Dalian, China

${ }^{e}$ Department of Heart Intensive Care Unit, The First Affiliated Hospital of Dalian Medical University, 193 Lianhe Road, Dalian 116011, China. E-mail: Yang060188@ 163.com; Fax: +86-411-83635963; Tel: +86-411-83635963 lipids and transition to foam cells. Macrophage-derived foam cells release cytokines that recruit more macrophages to lesions and influence lipid deposition. ${ }^{5}$ Therefore, similar pathogenic mechanisms may contribute to the progression of atherosclerosis and chronic kidney disease. However, the underlying pathophysiological mechanisms of the relationship between hypercholesterolaemia and kidney injury are not yet fully understood.

Recently, there has been a growing interest in using natural phytochemical compounds as treatment alternatives in several conditions including renal diseases. Indeed, it has been estimated that at least $25 \%$ of the drugs used over the past few decades have been directly derived from plants and approximately $25 \%$ are chemically altered natural products. ${ }^{6}$ Thymoquinone is one of these compounds. It is the main active ingredient of Nigella sativa, commonly known as black cumin or black seed, an annual flowering plant native to some areas such as the Mediterranean countries. ${ }^{7}$ Since its first extraction in $1963,{ }^{8}$ thymoquinone has been shown to act as a potent free radical and superoxide scavenger. ${ }^{9-11}$ In addition, it has been shown to have anti-inflammatory properties in vivo and vitro studies. $^{12,13}$ It has also been shown to exert positive effects on DOX-induced nephropathy and ischaemia-reperfusion-induced 
kidney injury in rats. ${ }^{14,15}$ However, the function of thymoquinone in hypercholesterolemia-induced renal injury is unclear.

\section{Materials and methods}

All animal procedures were performed in accordance with the Guidelines for Care and Use of Laboratory Animals of Dalian Medical University and approved by the Animal Ethics Committee of the First Affiliated Hospital of Dalian Medical University. $\mathrm{ApoE}^{-1-}$ mice were purchased from Beijing Vital River Lab animal technology Co., Ltd. (Beijing, China). All mice were housed in a room with a $12: 12$ hour light-dark cycle at $24^{\circ} \mathrm{C}$. At 8 weeks old, the male mice were randomly divided into the following three groups: $\mathrm{ApoE}^{-/-}$mice fed a normal diet $(n=7)$, a high-cholesterol diet $(n=7)$, or thymoquinone by gavaged $(25 \mathrm{mg}$ per $\mathrm{kg}$ per day; Sigma-Aldrich, St. Louis, MO, USA) + a high-cholesterol diet $(n=$ 7). The high-cholesterol diet contained $1.5 \%$ cholesterol and $15 \%$ fat. The experimental diet was purchased from the Shanghai Slac Laboratory Animal Co., Ltd. (Shanghai, China). Each group of mice were fed the corresponding diet for 8 weeks. Blood samples were obtained from the inferior vena cava and collected in serum tubes; the samples were then stored at $-80{ }^{\circ} \mathrm{C}$ until use. Coronal sections of kidneys were fixed in $10 \%$ formalin and then embedded in paraffin for histological evaluation or embedded in OCT compound (Torrance, CA, USA) and stored at $-80^{\circ} \mathrm{C}$ for Oilred $\mathrm{O}$ staining. The remainder of the kidney was snap-frozen in liquid nitrogen for mRNA or immunoblotting analysis. All animal experiments were performed in accordance with the Guide for the Care and Use of Laboratory Animals. The study was approved by the ethical committee of the First Affiliated Hospital of Dalian Medical University.

\section{Biochemical measurements}

Serum was obtained and stored at $-80{ }^{\circ} \mathrm{C}$. Total cholesterol, triglycerides, and low-density lipoprotein cholesterol (LDL-c) were measured using a Hitachi 7020 automatic analyser (Hitachi, Tokyo, Japan).

\section{Morphologic analysis and immunohistochemistry}

Kidney samples were collected and either fixed in $4 \%$ paraformaldehyde or snap frozen in liquid nitrogen. Samples were embedded in paraffin or OCT and were cut into slices using a microtome (Leica RM 2235 or Leica CM1850UV; Leica, Solms, Germany). The slices were then mounted onto glass slides, and histological examinations were performed. Frozen sections were used to evaluate lipid deposition using Oil-red O staining (Sigma, Santa Clara, CA, USA). Immunohistochemistry was performed using Histone Simple stain kits (Nichirei, Tokyo, Japan) according to the manufacturer's instructions. Briefly, paraffin-embedded sections were deparaffinised with xylene and then rehydrated in a descending series of ethanol. The sections were treated for 15 min with $3 \% \mathrm{H}_{2} \mathrm{O}_{2}$ in methanol to inactivate endogenous peroxidases and were then incubated at room temperature for 1 hour with primary antibodies against CD68 (rabbit anti-CD68 antibody, 1:500; Abcam, England) or LOX-1 (rabbit anti-LOX-1 antibody, 1:200; Abcam). All sections were observed under an Olympus $\mathrm{B} \times 40$ upright light microscope (Olympus, Tokyo, Japan). We quantified positive area using NIH ImageJ software (http://rsbweb.info.nih.gov/ij/). ${ }^{16}$

\section{RNA isolation and real-time PCR}

Total RNA was isolated from glomeruli using ISOGEN (Nippon gene, Tokyo, Japan) according to the manufacturer's protocol. Complementary DNA (cDNA) was synthesized from total RNA using a first-strand cDNA synthesis kit (SuperScript VILO cDNA Synthesis Kit; Life Technologies, Carlsbad, CA, USA) according to the manufacturer's protocol. Gene expression was quantitatively analysed by real-time PCR using fluorescent SYBR Green technology (Light Cycler; Roche Molecular Biochemicals). $\beta$ actin cDNA was amplified and quantitated with each cDNA sample to normalize the relative amounts of target genes. The primer sequences are listed in Table 1.

\section{Western blotting of kidney tissue}

Proteins were extracted from renal cortical tissues using radioimmunoprecipitation assay buffer (P0013B; Beyotime, Shanghai, China). The samples were electrophoresed on $10 \%$ SDS-PAGE gels, and proteins were transferred to polyvinylidene fluoride membranes (Immobilon, Millipore, Billerica, MA, USA). The membranes were blocked in Tris-buffered saline with $0.1 \%$ Tween-20 (TBS-T) containing 5\% skim milk and then incubated in primary antibody diluents (P0023A; Beyotime) and gently shaken overnight at $4{ }^{\circ} \mathrm{C}$. Primary antibodies against LOX-1 (rabbit anti-LOX-1 antibody, 1:250; Abcam), phospho-ERK (rabbit anti-phospho-ERK, 1:1000; Cell Signalling Technology), and $\beta$-actin (1:1000; Cell Signalling Technology) were used. The membranes were then incubated with the secondary antibody (anti-rabbit Ig-G, 1:1000; Cell Signalling Technology) for 1 hour. This analysis was independently performed three times. The protein levels are expressed as protein/ $\beta$-actin ratios to minimize loading differences. The relative signal intensity was quantified using NIH ImageJ software.

Table 1 Primer oligonucleotide sequences ${ }^{a}$

\begin{tabular}{ll}
\hline Gene & Primers \\
\hline \multirow{2}{*}{ LOX-1 } & F:5'-CAAAGTCTCCCAACCAACCTGCAA-3' \\
& R:5'-ACATCCTGTCTTTCATGCGGCAAC-3' \\
SRA1 & F:5'-GTTAAAGGTGATGGGGGACA-3 \\
& R:5'-TCCCCTTCTCTCCCTTTTGT-3' \\
CD36 & F:5'-CCTTAAAGGAATCCCCGTGT-3' \\
& R:5'-TGCATTTGCCAATGTCTAGC-3' \\
ABCA1 & F:5'-AGCCAGAAGGGAGTGTCAGA-3' \\
& R:5'-CATGCCATCTGGGTAAACCT-3' \\
TNF- $\alpha$ & F:5'-TCTCATGCACCACCATCAAGGACT-3' \\
& R:5'-ACCACTCTCCCTTTGCAGAACTCA-3' \\
IL-6 & F:5'-TACCAGTTGCCTTCTTGGGACTGA-3' \\
& R:5'-TAAGCCTCCGACTTGTGAAGTGGT-3' \\
$\beta$-actin & F:5'-CGATGCCCTGAGGGTCTTT-3' \\
& R:5'-TGGATGCCACAGGATTCCAT-3'
\end{tabular}

${ }^{a}$ Abbreviations: LOX-1, lectin-like oxidized low-density lipoprotein receptor-1; SRA, scavenger receptor-A; ABCA1, ATP-binding cassette transporter A1; TNF- $\alpha$, tumor necrosis factor- $\alpha$; IL-6, interleukin-6. 


\section{Statistical analysis}

All data were presented as the mean \pm SEM. Statistical analysis was performed using SPSS software version 23.0 (SPSS Inc., Chicago, IL, USA). Intergroup variation was measured using one-way ANOVA and subsequent Tukey's test. The minimal level for statistical significance was set at $P<0.05$.

\section{Results}

\section{Metabolic characteristics}

The metabolic characteristics of $\mathrm{ApoE}^{-1-}$ mice after 8 weeks of dietary treatment are summarized in Table 2. The HD group showed a marked increase in total cholesterol and non-highdensity lipoprotein cholesterol levels in $\mathrm{ApoE}^{-/-}$mice, but these parameters were significantly decreased in the HD + TQ group. There was no difference between the HD + TQ and ND groups. Kidney/body weight ratio did not differ among the three groups.

\section{Thymoquinone reduced renal lipid accumulation in $\mathrm{ApoE}^{-/-}$ mice in the HD group}

We used Oil-red O staining to evaluate renal lipid accumulation. We detected increased lipid retention in the kidneys of $\mathrm{ApoE}^{-1-}$ mice in the HD group. Interestingly, the mice in the HD + TQ group displayed markedly reduced renal lipid deposition compared with the $\mathrm{ApoE}^{-1-}$ mice despite the consumption of HD (Fig. 1A and B).

Table 2 Metabolic data from the three groups after 8 weeks of different treatment ${ }^{a}$

\begin{tabular}{|c|c|c|c|}
\hline & $\mathrm{ApoE}^{-1-} \mathrm{ND} n=7$ & $\operatorname{ApoE}^{-1-} \mathrm{HD} n=6$ & $\mathrm{ApoE}^{-1-} \mathrm{HD}+\mathrm{TQ} n=7$ \\
\hline Body weight (g) & $27.32 \pm 1.52$ & $29.47 \pm 1.95$ & $28.63 \pm 1.83$ \\
\hline Kidney/body weight ratio $\left(\mathrm{mg} \mathrm{g}^{-1}\right)$ & $6.44 \pm 0.95$ & $7.22 \pm 0.85$ & $6.53 \pm 1.05$ \\
\hline T-cholesterol $\left(\mathrm{mg} \mathrm{dl}^{-1}\right)$ & $425.03 \pm 32.16^{*}$ & $1862.67 \pm 136.32$ & $686.53 \pm 54.31 *$ \\
\hline Non-HDL-c $\left(\mathrm{mg} \mathrm{dl}^{-1}\right)$ & $127.43 \pm 26.72^{*}$ & $679.32 \pm 85.16$ & $218.47 \pm 23.96^{*}$ \\
\hline
\end{tabular}

${ }^{a}$ Abbreviations: TC, total cholesterol; HDL-c, high-density lipoprotein cholesterol. Data are means \pm SEM; $n=6-7$ per group. ${ }^{*} P<0.05$ vs. ApoE ${ }^{-/-}$ HD.

A

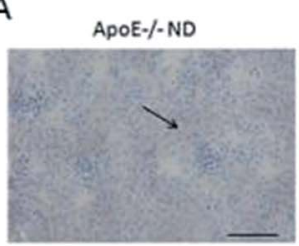

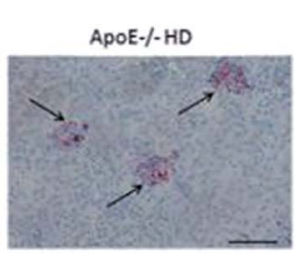

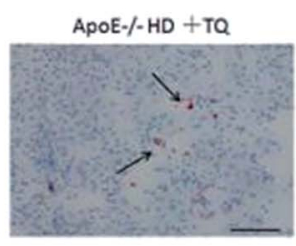

B

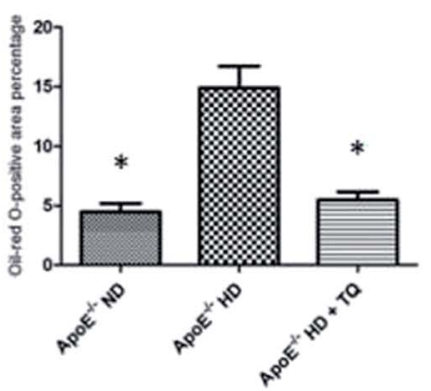

Fig. 1 Renal lipid accumulation in the three groups after 8 weeks with different diets. (A) Representative Oil-red O staining in the kidney tissue. Red: Oil-red O-positive cells, and blue: haematoxylin counterstaining. Scale bar $=200 \mu \mathrm{m}$. (B) Bar graph showing oil red O-positive cells in the kidney tissues. Data are means $\pm \mathrm{SEM} ; n=4$ in each group. $* P<0.05$.

A

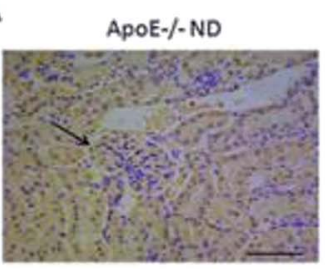

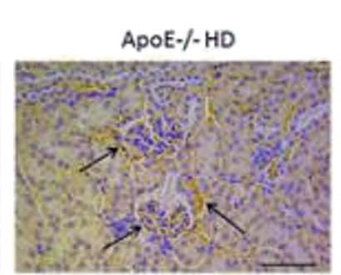

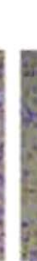

ApoE-/ $-\mathrm{HD}+\mathrm{TQ}$

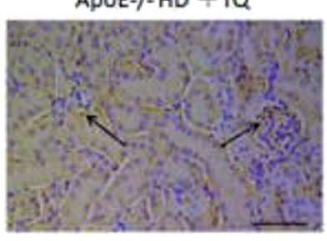

B

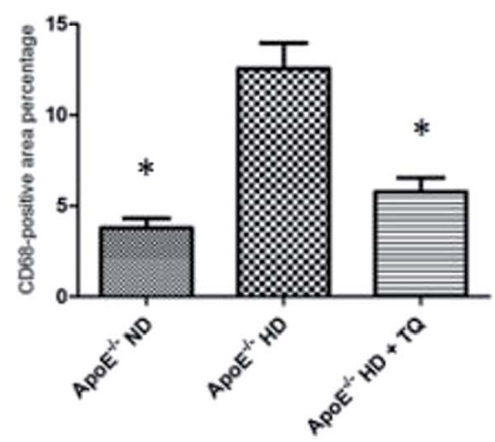

Fig. 2 CD68 expression in the kidney tissues of the three groups after 8 weeks with different diets. (A) Representative immunohistochemistry of CD68 expression in kidney tissues. Scale bar $=200 \mu \mathrm{m}$. Arrows indicate positively stained cells. (B) Bar graph summarizing CD68 expression in kidney tissues. Each bar represents the mean $\pm \mathrm{SEM} ; n=4$ in each group. ${ }^{*} P<0.05$. 
A
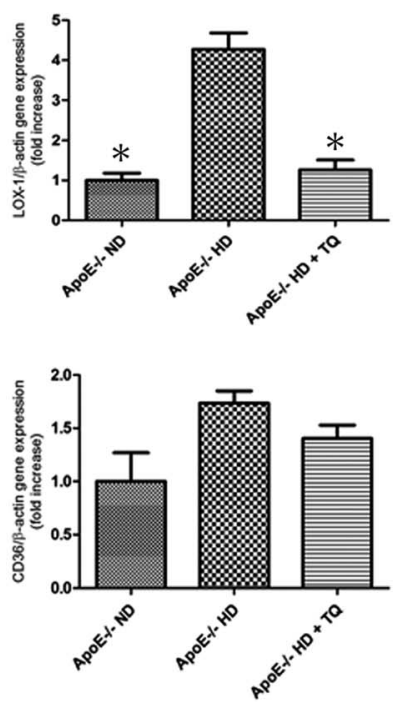

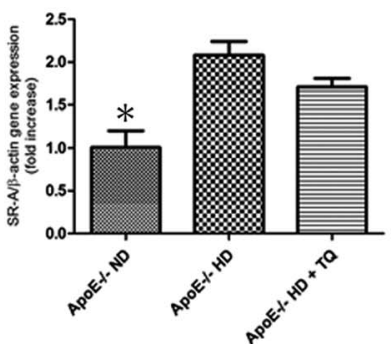

B

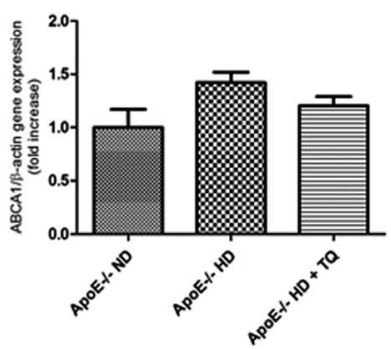

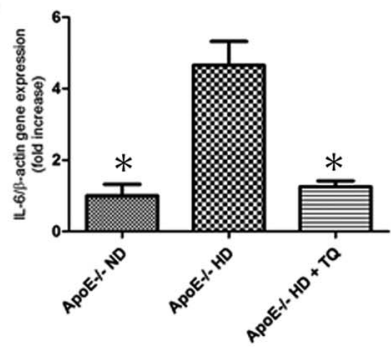

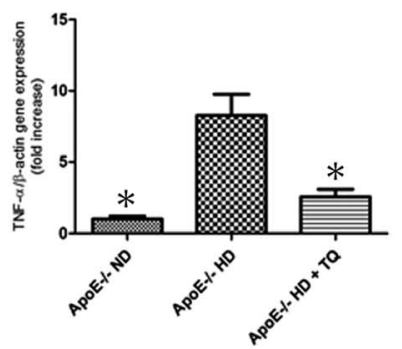

Fig. 3 Expression of scavenger receptors and pro-inflammatory genes in the kidney tissues of the three groups after 8 weeks with different diets. (A) Relative mRNA expression of LOX-1, SRA, CD36 and ABCA1 in the kidney tissues of the three groups after 8 weeks with different diets. (B) Relative mRNA expression of IL- 6 and TNF- $\alpha$ in the kidney tissues of the three groups after 8 weeks with different diets. Data are given as the means $\pm \mathrm{SEM} ; n=6$ in each group. $* P<0.05 \mathrm{vs}$. $\mathrm{ApoE}^{-1-} \mathrm{HD}$.

\section{Thymoquinone reduced macrophages in the kidneys of} $\mathrm{ApoE}^{-1-}$ mice in the HD group

To detect infiltrating macrophages, immunohistochemical analysis was performed against CD68 staining (Fig. 2A). The mice in the HD + TQ group showed markedly reduced CD68-positive staining in kidney tissues compared with $\mathrm{ApoE}^{-1-} \mathrm{HD}$ mice (Fig. 2B). These results indicate that thymoquinone reduced macrophage infiltration in the $\mathrm{ApoE}^{-/-} \mathrm{HD}$ mouse kidneys.

\section{Thymoquinone reduced LOX-1 gene expression in the kidneys of $\mathrm{ApoE}^{-/-}$mice fed HD}

To investigate the mechanism of lipid accumulation in the kidneys, gene expression of relevant receptors and the ATPbinding cassette transporter A1 (ABCA1) was examined in kidney tissues using real-time PCR. LOX-1 gene expression was significantly increased in the kidney tissues of the HD group compared with that in the ND group. The increased expression
A

ApoE-/-ND

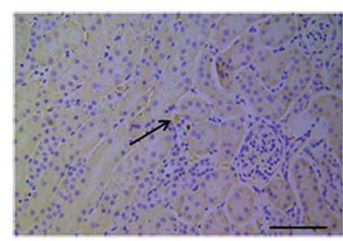

ApoE-/-HD

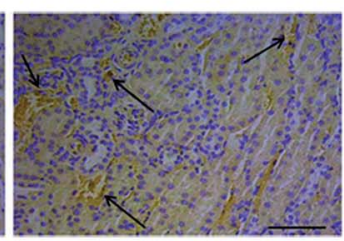

ApoE-/-HD+TQ

C

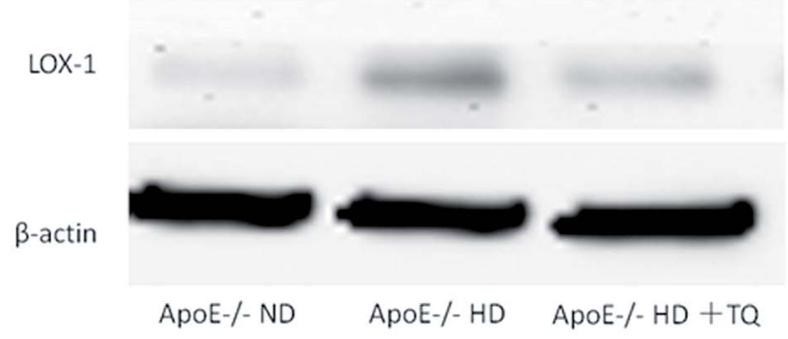

B

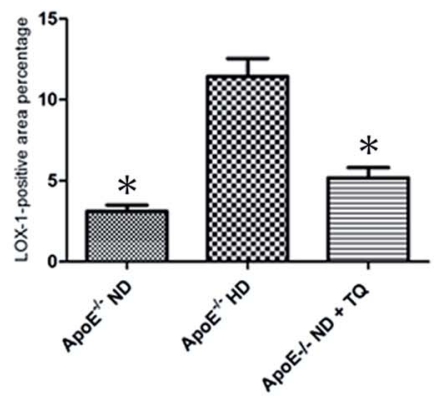

D

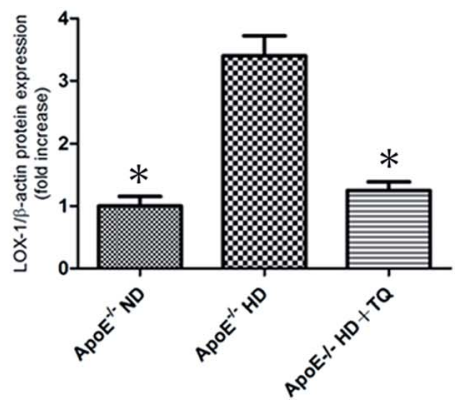

Fig. 4 LOX-1 expression in the kidney tissues of the three groups after 8 weeks with different diets. (A) Representative immunohistochemistry for LOX-1 in kidney tissues. Scale bar $=200 \mu \mathrm{m}$. Arrows indicate positively stained cells. (B) Bar graph summarizing LOX-1 expression in kidney tissues. (C) Immunoblotting for LOX-1 in kidney tissues. (D) Bar graph showing quantification of LOX-1 protein expression. Data are given as the means $\pm \mathrm{SEM} ; n=3-4$ in each group. ${ }^{*} P<0.05 \mathrm{vs}$. ApoE ${ }^{-1-} \mathrm{HD}$. 

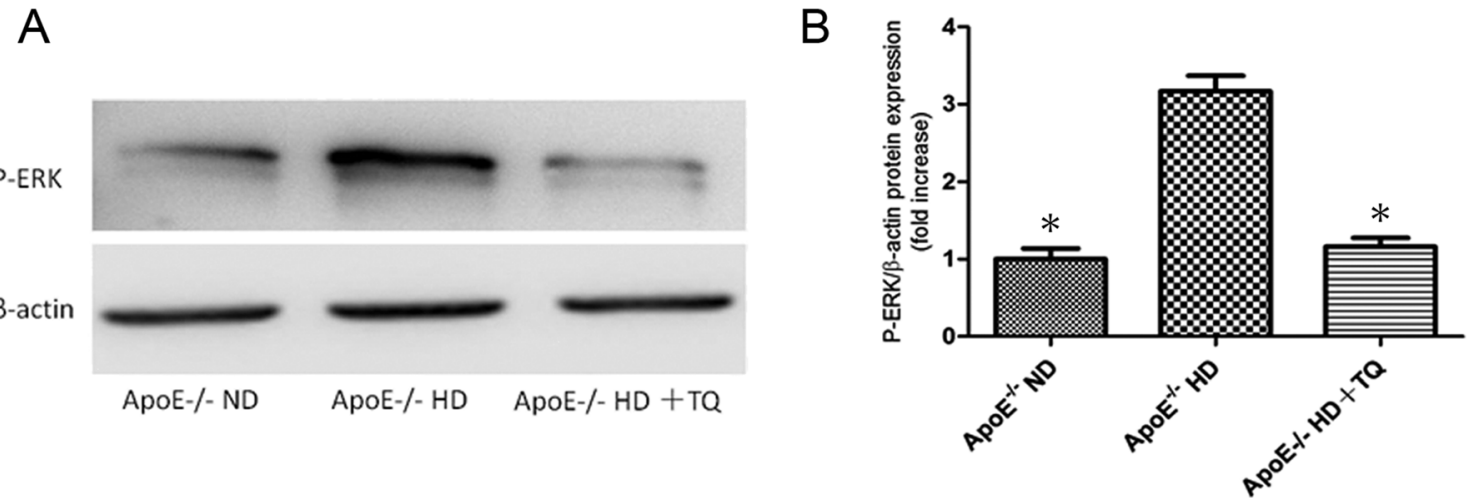

Fig. 5 Phospho-ERK protein expression in the kidney tissues of the three groups after 8 weeks with different diets. (A) Immunoblotting for phospho-ERK in kidney tissues. (B) Bar graph showing quantification of phospho-ERK protein expression. Data are given as the means $\pm S E M ; ~ n=$ 3 in each group. $* P<0.05$ vs. ApoE ${ }^{-1-} H D$.

of LOX-1 was suppressed in the HD + TQ group. The expression of scavenger receptor-class A (SR-A) and CD36 was increased in the HD group compared with that in the ND group; however, the levels were similar to those for the HD + TQ group. The expression of ABCA1 did not differ among the three groups (Fig. 3A). These results suggest that LOX-1, SR-A, and CD36 influence lipid accumulation in the kidney tissues of $\mathrm{ApoE}^{-/-}$ HD mice. LOX-1, in particular, appears to be a critical factor for mitigating lipid accumulation in the kidney tissue of $\mathrm{ApoE}^{-/-}$ $\mathrm{HD}+\mathrm{TQ}$ mice compared to that in the $\mathrm{ApoE}^{-1-} \mathrm{HD}$ mice.

\section{Thymoquinone reduced tumour necrosis factor (TNF)- $\alpha$ and interleukin (IL)-6 gene expression in the kidneys of $\mathrm{ApoE}^{-/-}$ mice fed HD}

To examine the involvement of pro-inflammatory cytokines in the gene expression of hypercholesteremic kidney tissues, the gene expression of IL- 6 and TNF- $\alpha$ was measured using realtime PCR (Fig. 3B). Both IL-6 and TNF- $\alpha$ were upregulated in the $\mathrm{ApoE}^{-/-} \mathrm{HD}$ mice; however, this upregulation was attenuated in $\mathrm{ApoE}^{-1-} \mathrm{HD}+\mathrm{TQ}$ mice.

\section{Thymoquinone reduced LOX-1 expression in the kidney tissues determined using immunohistochemistry and immunoblotting}

To evaluate LOX-1 expression in the kidney tissues, LOX-1 immunostaining was performed (Fig. 4A). The HD + TQ group had markedly reduced LOX-1 expression in kidney tissues compared to the HD group (Fig. 4B). Immunoblotting was performed for LOX-1 protein (Fig. 4C). We found that the LOX-1 protein expression was significantly suppressed in the HD + TQ group compared with that in the HD group (Fig. 4D). These results indicate that thymoquinone reduced LOX-1 expression in ApoE $^{-1-}$ HD mice.

Thymoquinone reduced the expression of phospho-ERK in the kidney tissues of ApoE $^{-/-}$HD mice

Protein kinases play a role in foam cell formation and lipid deposition, ${ }^{17}$ and immunoblotting for phospho-ERK protein was performed (Fig. 5A). We found that phospho-ERK was significantly suppressed in the HD + TQ group compared to that in the HD group (Fig. 5B).

\section{Discussion}

This study demonstrates that thymoquinone has a protective effect against progressive lipid deposition, macrophage accumulation and pro-inflammatory cytokine mRNA content in the kidney elicited by hypercholesterolemia.

The cholesterol in $\mathrm{ApoE}^{-/-}$mice is actually in LDL-C as well as in chylomicrons fractions. According to the metabolic characteristics, we found that TC and non-HDL-c were increased in the $\mathrm{HD}$ group compared with the $\mathrm{ND}$ group of $\mathrm{ApoE}^{-/-}$mice. These results are agreement with reports by Daniel Kolbus. ${ }^{18}$ Interestingly, TC and non-HDL-c were significantly suppressed in the HD + TQ group compared with that in the HD group. These results indicate that thymoquinone influences cholesterol metabolism, Shafeeque Ahmad et al. reported the same results and due to increased clearance rate of TG in hepatic $\beta$ VLDL secretion, ${ }^{\mathbf{1 9 , 2 0}}$ however, further studies are needed to clarify the mechanisms.

Hypercholesterolemia is a major independent risk factor for kidney disease. ${ }^{21}$ In hypercholesterolemia, cellular lipid homeostasis involves the regulation of influx, synthesis, catabolism, and efflux of lipids. An imbalance in these processes can result in the conversion of macrophages, mesangial cells, and vascular smooth muscle cells into foam cells. This process is mediated by several independent factors, including SR-A, class $B$ (CD36), and LOX-1, and it regulates the expression of the target gene ABCA1. ${ }^{22-24}$

Thymoquinone is a potent phytochemical anti-oxidant due to its scavenging activity against several ROS including superoxide anions, hydroxyl radicals and singlet molecular oxygen, and thus it can antagonize the adverse effects resulting from elevated ROS levels in various disorders. ${ }^{25,26}$ It has been shown that thymoquinone and tert-butylhydroquinone (TBHQ), a structurally related synthetic compound, can robustly inhibit iron-dependent microsomal lipid peroxidation. The anti- 
oxidative potential of thymoquinone may be related to the redox properties of its quinone moiety and its unrestricted ability to cross physiological barriers and access subcellular compartments, all of which help its radical scavenging effects. ${ }^{27,28}$

These effects include the reduction in blood total cholesterol and lipid peroxidation levels during kidney injury. ${ }^{14}$ Thymoquinone administration has been shown to protect kidneys from oxidative damage caused by pyelonephritis. In pyelonephritis, SOD and CAT activity and MDA levels are significantly abnormal; and the deregulation of these factors is resolved in pyelonephritis treated with thymoquinone. ${ }^{29}$

In our study, we analysed the gene expression of scavenger receptors including SR-A, CD36, and LOX-1. We found that LOX1 gene expression was supressed in the HD + TQ group. LOX-1 was originally identified in endothelial cells, and it is a $50 \mathrm{kDa}$ type II membrane glycoprotein that contains a short N-terminal cytoplasmic domain, a single transmembrane domain, a short neck or stalk region, and an ox-LDL-binding C-terminal extracellular C-type lectin-like domain. On the cell surface, LOX-1 consists of 3 homodimers that are bound to ox-LDL, and it plays an important role in ox-LDL uptake and foam cell formation. ${ }^{30,31}$ In contrast, deletion of LOX-1 has been shown to reduce the uptake of oxidized LDL and inhibit atherosclerosis in mice fed a high-cholesterol diet. ${ }^{32}$ Therefore, suppression of LOX-1 expression in $\mathrm{ApoE}^{-1-} \mathrm{HD}+\mathrm{TQ}$ mice may reduce foam cell formation. Thymoquinone also reduced LOX-1 protein expression in the kidney tissues of $\mathrm{ApoE}^{-/-} \mathrm{HD}$ mice. Protein kinases regulate foam cell formation and lipid deposition. As shown earlier, enhanced LOX-1 expression was attenuated by inhibitors of ERK, PKC and NF- $\mathrm{BB}$, indicating that the increased production of intracellular ROS and activation of the PKC/ MAPK pathway are the initial signalling events in LOX-1 gene regulation. ${ }^{16}$ Our results show that phospho-ERK expression was significantly suppressed in the HD + TQ group compared with that in the HD group. It is speculated that thymoquinone regulates LOX-1 via the phospho-ERK pathway.

Pro-inflammatory genes (TNF- $\alpha$ and IL-6) have been reported to be expressed at high levels and contribute to kidney injury in hyperlipidaemia. ${ }^{33-35}$ In contrast, TNF- $\alpha$ and IL-6 were shown to induce LOX-1 upregulation in smooth muscle cells. ${ }^{36}$ The present study shows that TNF- $\alpha$ and IL- 6 gene expression is reduced in the HD + TQ group compared with that in the HD group. Attenuation of TNF- $\alpha$ and IL- 6 expression may have also reduced LOX-1 expression in $\mathrm{ApoE}^{-1-} \mathrm{HD}+\mathrm{TQ}$ mice. It has been reported that thymoquinone reduces TNF- $\alpha$ and interleukin- 6 in blood and tissues and protects tissues by reducing inflammation. ${ }^{12,13}$ However, another reported that reduction of p-ERK with reduction in inflammation such as TNF- $\alpha .{ }^{37}$

Kidney injury induced by hyperlipidaemia is usually associated with an increase in the number of macrophages. Macrophage-derived foam cells release cytokines that recruit more macrophages to lesions and influence lipid deposition. ${ }^{5}$ The marker CD68 identifies macrophages. CD68-positive cells have been found to infiltrate glomeruli and interstitial lesions. ${ }^{38}$ As far as reported that LOX-1 increased with CD68 infiltration of atherosclerotic plaques in $\mathrm{ApoE}^{-1-}$ mice $^{39}$ and LOX-1 expression reduced with lower level of CD68 of high cholesterol diet induced mice. ${ }^{40}$ CD68 is a marker of pro-inflammatory M1, "classically activated" M1 macrophages produce proinflammatory cytokines such as TNF- $\alpha .^{41}$ In the present study, immunohistochemical staining with anti-CD68 antibody showed that CD68-positive cells were significantly increased in the HD group compared with that in the ND group of $\mathrm{ApoE}^{-/-}$ mice; however, mice in the HD + TQ group showed markedly reduced accumulation of CD68-positive cells in kidney tissues compared with mice in the HD group, similarly, with the TNF$\alpha$ gene expression. This indicates that thymoquinone reduces macrophage accumulation and TNF- $\alpha$ gene expression in mice in the HD group.

In conclusion, our data establish that thymoquinone contributes to the mitigation of hypercholesteraemic kidney injury as shown by the downregulation of LOX-1 and the suppression of foam cell formation, lipid deposition, and macrophage accumulation. These findings provide new insights into the role of thymoquinone in hypercholesterolemia-induced kidney injury and raise the possibility of a novel therapeutic intervention for treating the progression of chronic kidney disease.

\section{Author contributions}

Hongyang Liu designed this study; Guang Yang and Nan Li helped perform the experiments; Zuowei Pei, Yingshu Liu and Liyue Zhu analysed the data and interpreted the experimental results; Zuowei Pei prepared the figures and drafted the manuscript; and Hongyang Liu helped to revise the manuscript. All authors read and approved the final manuscript.

\section{Conflicts of interest}

The authors declare no conflict of interest.

\section{Acknowledgements}

This work was finally supported by the social development project of public welfare technology (No. 2015C33121).

\section{References}

1 J. A. Piedrahita, S. H. Zhang, J. R. Hagaman, P. M. Oliver and N. Maeda, Generation of mice carrying a mutant apolipoprotein $\mathrm{E}$ gene inactivated by gene targeting in embryonic stem cells, Proc. Natl. Acad. Sci. U. S. A., 1992, 89, 4471-4475.

2 Z. Pei, T. Okura, T. Nagao, D. Enomoto, M. Kukida, A. Tanino, K. Miyoshi, M. Kurata and J. Higaki, Osteopontin deficiency reduces kidney damage from hypercholesterolemia in Apolipoprotein E-deficient mice, Sci. Rep., 2016, 6, 28882.

3 X. Z. Ruan, Z. Varghese and J. F. Moorhead, An update on the lipid nephrotoxicity hypothesis, Nat. Rev. Nephrol., 2009, 5, 713-721.

4 M. Kuwahara, K. Bannai, H. Segawa, K. Miyamoto and H. Yamato, Cardiac remodeling associated with protein 
increase and lipid accumulation in early-stage chronic kidney disease in rats, Biochim. Biophys. Acta, 2014, 1842, 1433-1443.

5 M. P. De Winther and M. H. Hofker, Scavenging new insights into atherogenesis, J. Clin. Invest., 2000, 105, 1039-1041.

6 P. Vuorelaa, M. Leinonenb, P. Saikkuc, P. Tammelaa, J. P. Rauhad, T. Wennberge and H. Vuorela, Natural products in the process of finding new drug candidates, Curr. Med. Chem., 2004, 11, 1375-1389.

7 H. Gali-Muhtasib, A. Roessner and R. Schneider-Stock, Thymoquinone: a promising anti-cancer drug from natural sources, Int. J. Biochem. Cell Biol., 2006, 38, 1249-1253.

$8 \mathrm{M}$. El-Dakhakhny, Studies on the chemical constitution of Egyptian N. sativa L. seeds, Planta Med., 1963, 11, 465-470.

9 A. S. Awad, R. Kamel and M. A. Sherief, Effect of thymoquinone on hepatorenal dysfunction and alteration of CYP3A1 and spermidine/spermine N-1-acetyl-transferase gene expression induced by renal ischaemia-reperfusion in rats, J. Pharm. Pharmacol., 2011, 63, 1037-1042.

10 M. M. Farag, G. O. Ahmed, R. R. Shehata and A. H. Kazem, Thymoquinone improves the kidney and liver changes induced by chronic cyclosporine A treatment and acute renal ischaemia/reperfusion in rats, J. Pharm. Pharmacol., 2015, 67, 731-739.

11 M. A. Mansour, M. N. Nagi, A. S. El-Khatib and A. M. AlBekairi, Effects of thymoquinone on antioxidant enzyme activities, lipid peroxidation and DT-diaphorase in different tissues of mice: a possible mechanism of action, Cell Biochem. Funct., 2002, 20, 143-151.

12 I. Tekeoglu, A. Dogan and L. Demiralp, Effects of thymoquinone (volatile oil of black cumin) on rheumatoid arthritis in rat models, Phytother. Res., 2006, 20, 869-871.

13 F. Vaillancourt, P. Silva, Q. Shi, H. Fahmi, J. C. Fernandes and M. Benderdour, Elucidation of molecular mechanisms underlying the protective effects ofthymoquinone against rheumatoid arthritis, J. Cell. Biochem., 2011, 112, 107-117.

14 O. A. Badary, A. B. Abdel-Naim, M. H. Abdel-Wahab and F. H. Hamada, The influence of thymoquinone on doxorubicin-induced hyperlipidemic nephropathy in rats, Toxicology, 2000, 143, 219-226.

15 F. T. Hammad and L. Lubbad, The effect of thymoquinone on the renal functions following ischemia-reperfusion injury in the rat, Int. J. Physiol., Pathophysiol. Pharmacol., 2016, 4, 152-159.

16 A. Mehlem, C. E. Hagberg, L. Muhl, U. Eriksson and A. Falkevall, Imaging of neutral lipids by oil red $\mathrm{O}$ for analyzing the metabolic status in health and disease, Nat. Protoc., 2013, 6, 1149-1154.

17 L. Li, T. Sawamura and G. Renier, Glucose enhances human macrophage LOX-1 expression: role for LOX-1 in glucoseinduced macrophage foam cell formation, Circ. Res., 2004, 94, 892-901.

18 D. Kolbus, O. H. Ramos, K. E. Berg, J. Persson, M. Wigren, H. Björkbacka, G. N. Fredrikson and J. Nilsson, CD8+ T cell activation predominate early immune responses to hypercholesterolemia in Apoe $^{-/-}$mice, BMC Immunol., 2010, 11, 58.
19 S. Ahmad and Z. H. Beg, Elucidation of mechanisms of actions of thymoquinone-enriched methanolic and volatile oil extracts from Nigella sativa against cardiovascular risk parameters in experimental hyperlipidemia, Lipids Health Dis., 2013, 12, 86.

20 A. M. MacKinnon, J. Savage, R. A. Gibson and P. J. Barter, Secretion of cholesteryl ester-enriched very low density lipoproteins by the liver of cholesterol-fed rabbits, Atherosclerosis, 1985, 2, 145-155.

21 E. S. Schaeffner, T. Kurth, G. C. Curhan, R. J. Glynn, K. M. Rexrode, C. Baigent, J. E. Buring and J. M. Gaziano, Cholesterol and the risk of renal dysfunction in apparently healthy men, J. Am. Soc. Nephrol., 2013, 14, 2084-2091.

22 C. K. Abrass, Cellular lipid metabolism and the role of lipids in progressive renal disease, Am. J. Nephrol., 2004, 24, 46-53.

23 C. K. Glass and J. L. Witztum, Atherosclerosis: the road ahead, Cell, 2001, 104, 503-516.

24 A. M. Vaughan, C. Tang and J. F. Oram, ABCA1 mutants reveal an interdependency between lipid export function, apoA-I binding activity, and Janus kinase 2 activation, $J$. Lipid Res., 2009, 50, 285-292.

25 M. A. Mansour, M. N. Nagi, A. S. El-Khatib and A. M. AlBekairi, Effects of thy-moquinone on antioxidant enzyme activities, lipid peroxidation and DT-diaphorase in different tissues of mice: a possible mechanism of action, Cell Biochem. Funct., 2002, 20, 143-151.

26 M. N. Nagi and M. A. Mansour, Protective effect of thymoquinone against doxorubicin-induced cardiotoxicity in rats: a possible mechanism of protection, Pharmacol. Res., 2000, 41, 283-289.

27 O. A. Badary, R. A. Taha, A. M. Gamal El-Din and M. H. Abdel-Wahab, Thymoquinoneis a potent superoxide anion scavenger, Drug Chem. Toxicol., 2003, 26, 87-98.

28 M. H. Daba and M. S. Abdel-Rahman, Hepatoprotective activity of thymoquinonein isolated rat hepatocytes, Toxicol. Lett., 1998, 95, 23-29.

29 O. Evirgen, A. Gokce, O. H. Ozturk, E. Nacar, Y. Onlen and B. Ozer, Effect of thymoquinone on oxidative stress in Escherichia coli-induced pyelonephritisin rats, Current Therapeutic Research, Clinical and Experimental, 2011, 72, 204-215.

30 T. Sawamura, N. Kume, T. Aoyama, H. Moriwaki and H. Hoshikawa, An endothelial receptor for oxidized lowdensity lipoprotein, Nature, 1997, 386, 73-77.

31 S. Gao and Y. J. Geng, LOX-1: a male hormone-regulated scavenger receptor for atherosclerosis, Vasc. Pharmacol., 2013, 59, 138-143.

$32 \mathrm{C}$. $\mathrm{Hu}$, LOX-1 deletion decreases collagen accumulation in atherosclerotic plaque in low-density lipoprotein receptor knockout mice fed a high-cholesterol diet, Cardiovasc. Res., 2008, 79, 287-293.

33 W. Wang, B. He, W. Shi, X. Liang, J. Ma, Z. Shan and Z. Hu, Deletion of scavenger receptor A protects mice from progressive nephropathy independent of lipid control during diet-induced hyperlipidemia, Kidney Int., 2102, 81, 1002-1014. 
34 A. Ambrosch, R. Muller, C. Freytag, S. Borgmann, J. Kraus and J. Dierkes, Small-sized low-density lipoproteins of subclass B from patients with end-stage renal disease effectively augment tumor necrosis factor- $\alpha$-induced adhesive properties in human endothelial cells, Am. J. Kidney Dis., 2002, 39, 972-984.

35 M. Tomiyama-Hanayama, Effect of interleukin-6 receptor blockage on renal injury in apolipoprotein E-deficient mice, Am. J. Physiol., 2009, 297, 679-684.

36 O. Hofnagel, B. Luechtenborg, K. Stolle, S. Lorkowski and H. Eschert, Proinflammatory cytokines regulate LOX-1 expression in vascular smooth muscle cells, Arterioscler., Thromb., Vasc. Biol., 2004, 24, 1789-1795.

37 L. Liu, J. Ren, Z. He, et al., Cholesterol-modified Hydroxychloroquine-loaded Nanocarriers in Bleomycininduced Pulmonary Fibrosis, Sci. Rep., 2017, 7, 10737.
38 S. Guo, T. A. Wietecha, K. L. Hudkins, Y. Kida and M. W. Spencer, Macrophages are essential contributors to kidney injury in murine cryoglobulinemic membranoproliferative glomerulonephritis, Kidney Int., 2011, 9, 946-958.

39 M. Zhang, H. Zhao, J. Cai, et al., Chronic administration of mitochondrion-targeted peptide SS-31 prevents atherosclerotic development in ApoE knockout mice fed Western diet, PLoS One, 2017, 12, e0185688.

40 J. L. Mehta, N. Sanada, C. P. Hu, et al., Deletion of LOX-1 reduces atherogenesis in LDLR knockout mice fed high cholesterol diet, Circ. Res., 2007, 11, 1634-1642.

41 F. O. Martinez, S. Gordon, M. Locati, et al., Transcriptional profiling of the human monocyte-to-macrophage differentiation and polarization: new molecules and patterns of gene expression, J. Immunol., 2006, 177, 73037311. 